\title{
SPINAL BLOCKS WITH AND WITHOUT MORPHINE IN WOMEN UNDERGOING HYSTERECTOMIES- A RANDOMIZED STUDY
}

\author{
Marion E K, Krystyna Hansen, Gunilla E Tegerstedt ${ }^{1}$, Christer H. Svensen ${ }^{2}$, \\ Audrius Andrijauskas ${ }^{3}$, Dan Drobin ${ }^{4}$ \\ Karolinska Institutet, Department of Clinical Science and Education, Department \\ of Obstetrics and Gynecology, Södersjukhuset, Stockholm, Sweden ${ }^{1}$, Head, \\ Research and Education, Karolinska Institutet, Department of Clinical Science \\ and Education, Section of Anesthesiology and Intensive Care, Södersjukhuset, \\ Stockholm, Sweden ${ }^{2}$, Vilnius University Hospital, Section of Anaesthesiology and \\ Intensive Care ${ }^{3}$, Department of Anesthesiology, Linköping University Hospital, \\ Linköping, Sweden ${ }^{4}$ \\ *Corresponding author: marion.ek@sodersjukhuset.se
}

Keywords: hysterectomy, pain, nausea, intrathecal, patient-controlled analgesia

\section{Objective:}

To study whether a group of patients going through abdominal hysterectomies under general anesthesia could reduce their time of discharge and consumption of parenteral opioids by adding morphine to an intrathecal block.

\section{Methods:}

Sixty seven healthy women, undergoing abdominal hysterectomies. Patients had general anesthesia in combination with spinal blocks. Randomization between intrathecal blocks with morphine (group 1) and without morphine (group 2), in combination with either postoperative intermittent (group 1) or patient controlled analgesia (group 2).

\section{Results:}

The median time to discharge in group 1 was 53 hours and in group two 69 hours. Time of discharge was not statistically significant $(\mathrm{p}=0.51)$. The patients in group 2 had significantly higher visual analogue scores for pain postoperatively. More postoperative opioids were also consumed in that group $(\mathrm{p}<0.0001)$. Furthermore, significantly more patients in group 2 compared to group 1 indicated that they had suffered from major nausea $(\mathrm{p}=0.01)$.

\section{Conclusion:}

In summary, we conclude that intrathecal block with morphine in combination with postoperative nurse administered opioids did not show a difference in time to discharge compared to intratechal block without morphine in combination with PCA. However, there was a significant reduction in the level of pain and nausea as well as consumption of postoperative opioids. Based on the questionnaire, significantly more patients suffered from major nausea in the group without intrathecal morphine.

In order to facilitate earlier discharge after surgical procedures it is important that patients do not suffer from postoperative pain, nausea or vomiting
(PONV) [1] [2] [3] [4]. General anesthesia for abdominal hysterectomies can be supplemented by an intrathecal block for postoperative pain relief[5]. This is normally given as a local 
anesthetic in combination with an opioid[6]. Although fentanyl is widely used per-operatively,

\begin{tabular}{|l|l|l|l|}
\hline $\begin{array}{l}\text { Characteristic } \\
\text { s }\end{array}$ & $\begin{array}{l}\text { Group 1 } \\
\mathbf{n}=35\end{array}$ & $\begin{array}{l}\text { Group 2 } \\
\mathbf{n}=33\end{array}$ & $\mathbf{P}$ \\
\hline Age (yr) & $45 \pm 4$ & $48 \pm 6$ & 0.077 \\
\hline Height (cm) & $166 \pm 7$ & $167 \pm 7$ & 0.349 \\
\hline Weight (kg) & $67 \pm 8$ & $72 \pm 14$ & 0.317 \\
\hline $\begin{array}{l}\text { Body mass } \\
\text { index (kg.m2) }\end{array}$ & $24 \pm 3$ & $25 \pm 4$ & 0.278 \\
\hline ASA 1/2/3 (n) & $29 / 6 / 0$ & $18 / 14 / 1$ & \\
\hline Smokers (\%) & 17 & 21 & 0.98 \\
\hline $\begin{array}{l}\text { Duration of } \\
\text { surgery (min) }\end{array}$ & $95 \pm 24$ & $105 \pm 33$ & 0.329 \\
\hline Bleeding (ml) & $373 \pm 300$ & $352 \pm 286$ & 0.622 \\
\hline $\begin{array}{l}\text { Type of incision } \\
\text { (P/M) }\end{array}$ & $30 / 5$ & $26 / 7$ & \\
\hline
\end{tabular}

morphine would be more preferable due to its longer duration. However, morphine has some possible side-effects that needs to be addressed[7]. Postoperatively, pain control is conventionally administered by either intermittent or patient controlled analgesia regimens (PCA) [8]. Opioids are excellent pain controllers, however, particularly in abdominal hysterectomies, PCA is attributed to a higher level of PONV[6].

The aim of this study was to compare whether intrathecal blocks with or without added morphine could make a difference as regards to time to discharge, level of nausea and vomiting and consumption of postoperative opioids.

\section{Materials and Methods}

This study was designed as a single center, prospective and randomized study. The study was approved by the regional board of the Ethics Committee at Karolinska Institutet in Stockholm. Sixty seven women, with mean age 46 years (range 36-62) were included from September 2005 to February 2007. Written informed consent was obtained from each patient. Patients who underwent a total abdominal hysterectomy due to a benign cause were included. Patients suffering from cancer were not included. All patients were classified as either I, II or III according to the American Society of Anesthesiologists (ASA) classification. Further exclusion criteria were obesity (body mass index $>30 \mathrm{~kg} / \mathrm{m}^{2}$ ) and any contraindication to a spinal puncture. The patients were monitored 8-10 hours postoperatively in the PACU unit and since this unit closed 1600 it was only possible to perform the study on one patient each day. Normally, this meant that the study patient had to be the first patient on the schedule.

One hundred and seventy nine patients were eligible. Of these 82 patients 15 declined, the main reason being their reluctance to have a spinal puncture. See Figure 1.

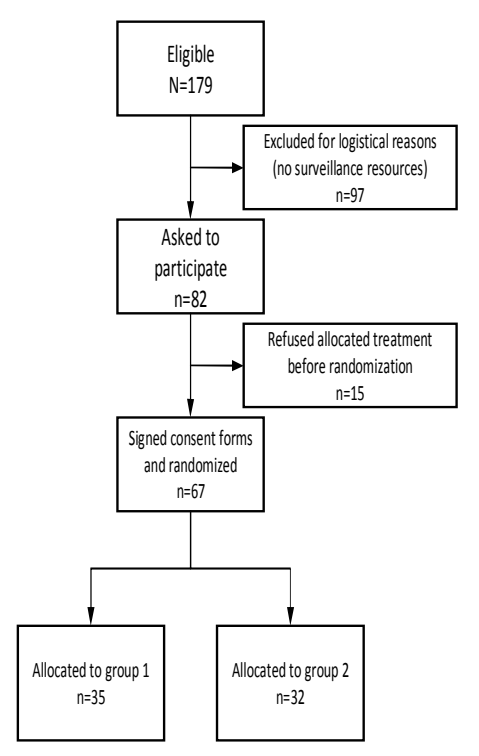

Fig. 1. The randomized trial flow diagram, including total sample size, enrollment and intervention allocation.

Patient characteristics are shown in Table.

Table.Patient characteristics at baseline. Values expressed as mean \pm SD. Group 1= Intrathecal morphine. Group 2= Intrathecal without morphine. $\mathrm{P}=$ Pfannenstiel. M=Midline. Groups were compared using the rank sum test for continuous variables.

Before surgery, all patients received instructions on how to use the visual analogue scale (VAS), graded from 0 (no pain or no nausea) to 100 (worst pain or worst nausea imaginable). The patients were randomly allocated by a computer-generated list (SPSS) into two groups; either to receive intrathecal (IT) morphine $(0.2 \mathrm{mg})$ combined with 
fentanyl $(10 \mu \mathrm{g})$ and bupivacaine $(12.5 \mathrm{mg})(\mathrm{n}=34$, group 1). The other group received intrathecal fentanyl only $(10 \mu \mathrm{g})$ and bupivacaine $(12.5 \mathrm{mg})$ $(n=33$, group 2). All patients received paracetamol $1 \mathrm{~g}$ orally as pre-medication one hour before surgery. Spinal anesthesia was then performed using a standardized technique with a (25-G Whitacre) spinal needle at the L2-L3 or L3-L4 inter-space. All patients received $50 \mu \mathrm{g}$ of fentanyl prior to induction. The general anesthetic was standardized using propofol 2-3 $\mathrm{mg} / \mathrm{kg}$. Atracurium 0.3-0.6 mg/kg was given prior to tracheal intubation. Anesthesia was maintained with sevoflurane at a level of $0.7-0.9$ on the vaporizer. The $\mathrm{O} \square$ and $\mathrm{N} \square \mathrm{O}$ fractions were 0.35 $\%$ and $0.65 \%$ respectively.

For postoperative pain control the following regimens were used:

Both groups were orally administered paracetamol tablets, $1 \mathrm{~g}$, four times daily together with diclofenac $50 \mathrm{mg}$ three times daily unless this was contraindicated. Group 1 was given intravenous morphine 1-5 mg upon patient request administered by the attending nurse.

In the recovery unit the patients in group 2 initially received a titrated dose of morphine $(2-5 \mathrm{mg}$ boluses at ten-minute intervals) to obtain pain relief. After this initial stage further intravenous analgesia was administered by the patient via a disposable patient controlled analgesia device (PCA) (Gemstar®, Abbot). The PCA pump was programmed to deliver $1 \mathrm{mg}$ ketobemidone boluses with a six-minute lockout interval and no maximum dose. Postoperative nausea and vomiting were treated with ondansetron $4 \mathrm{mg}$ intravenously (Zofran ${ }^{\circledR}$, Glaxo Wellcome). Pain at rest and during coughing and nausea was measured at 3, 6 and 10 hours after surgery and also at 10 AM the day after surgery by a trained attending nurse. Before discharge, all patients were requested to answer an inquiry form with three questions with yes/no alternatives. The questions were:

1/ Have you received enough analgesia after your surgery?

2/ Have you experienced much nausea after your surgery?

3/ Would you recommend the method of analgesia you received to a close friend?
The primary outcome parameter to be tested was the difference in time to discharge between the groups. Criteria for discharge of the patient were: oral pain medication only, no fever, adequate mobilization (the patient should be able to shower, fetch a food tray, walk the corridor and have no indwelling urinary catheter)

Secondary outcomes were differences in VAS score for pain and nausea differences in opioid consumption and differences in answers to the inquiry form.

\section{Power calculation}

The earliest theoretically possible time of discharge was at 3 PM the day after surgery when the afternoon rounds take place. We know by experience that most patients with general anaesthesia and no spinal analgesia are eligible for discharge at 8 am on the second day after surgery. The difference between the theoretically earliest time of discharge and this time point is 17 hours which was the difference that the study was designed to discover. With a power of $80 \%$ using a t-test and two-tailed significance level (alpha) 5\%, we needed 33 patients in each group. The power analysis was based on independent samples with a normal distribution and an assumed standard deviation in each group of 22 hours.

\section{Statistics}

All statistical analyses were carried out using the Statistical Package for the Social Sciences (2008, SPSS Inc.Chicago, IL, USA). Due to skew ness of data, we used the Mann-Whitney $U$ test to compare the median differences in time to discharge, ketobemidone requirement and the occurrence of pain and nausea between $. \mathrm{P}<0.05$ was considered significant.

\section{Results}

The median time to discharge in group 1 was 53 (24-172) hours and 69 hours (46-99) in group 2. However, this difference was not statistically significant $(\mathrm{p}=0.51)$. Significantly more ketobemidone was used in group 2 (mean $44 \mathrm{mg}$ \pm 19 ) than in group 1 (mean $12 \mathrm{mg} \pm 19$ ) ( $\mathrm{p}<0.0001$ ). See Figure 2. 


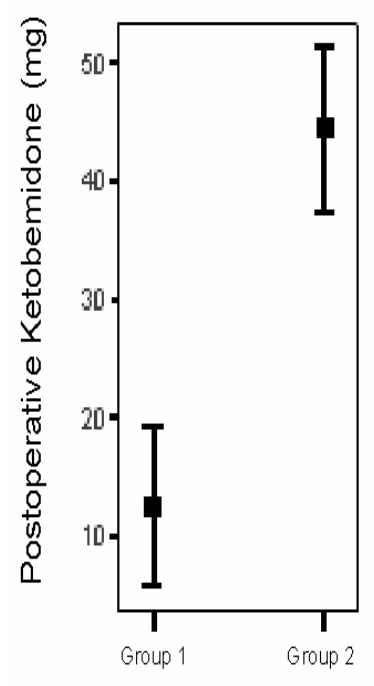

Fig 2. Consumption of ketobemidone.

Mean ketobemidone consumption (mg) in the two groups after surgery. Error bars show 95\% CI of the mean. $(\mathrm{p}<0.0001)($ Mann-Whitney).

The patients in group 2 had significantly higher VAS scores for pain, both at rest and while coughing. This occurred at all checkpoints $(3,6$, 10 hours after completed surgery and at 10 AM the day after) compared to the patients in group 1 (Figure 3 A-B).

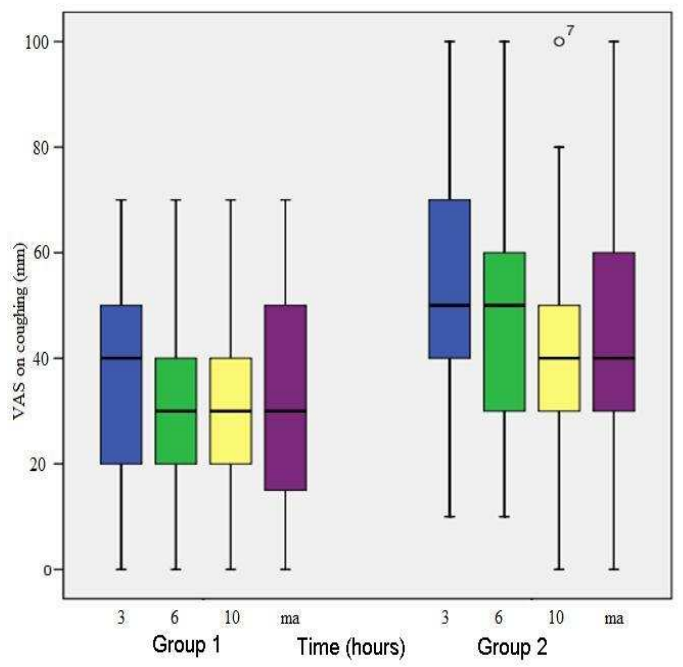

Fig. 3A. Pain relief while coughing after abdominal hysterectomy.

Box plot of pain scores (VAS) while coughing after abdominal hysterectomy in the intrathecal with morphine (group 1, left side) and in the intrathecal without morphine (group 2, right side).
The open circle represents an outlier. ma= morning after the first postoperative day 10 a.m. Pain scores are higher in group 2 than in group 2 during the first postoperative day $(\mathrm{t}=3 \mathrm{p}=0.005$, $\mathrm{t}=6 \mathrm{p}=0.001, \mathrm{t}=10 \mathrm{p}=0.01 \mathrm{t}=\mathrm{ma} \mathrm{p}=0.02$ ) (MannWhitney).

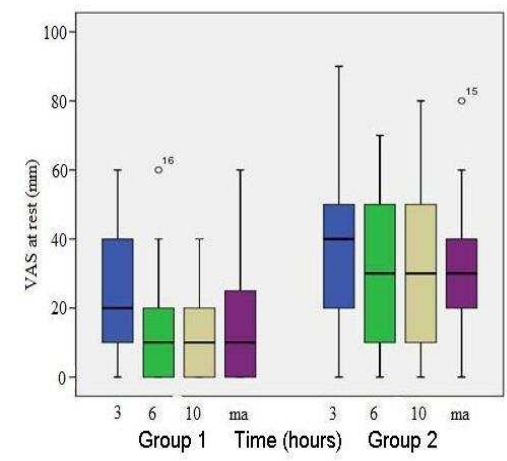

Fig 3B. Pain relief at rest after abdominal hysterectomy.

Box plot of pain scores (VAS) at rest after abdominal hysterectomy in the intrathecal with morphine (group 1, left side) and in the intrathecal without morphine ( group 2, right side). Open circles represent outliers. ma $=$ morning after the first postoperative day 10 a.m. Pain scores are higher in group 2 than in group 1 during the first postoperative day $(\mathrm{t}=3 \mathrm{p}=0.037, \mathrm{t}=6 \mathrm{p}=0.001, \mathrm{t}=10$ $\mathrm{p}=0.002 \mathrm{t}=$ ma $\mathrm{p}=0.008$ ) (Mann-Whitney).

VAS score for nausea was recorded at the same time as VAS pain scores. Initially there was no difference in VAS scoring for nausea between the groups. However, there was a diverging trend with increasing nausea in group 2 compared to group 1 reaching significant difference the morning after surgery. See Figure 4. 


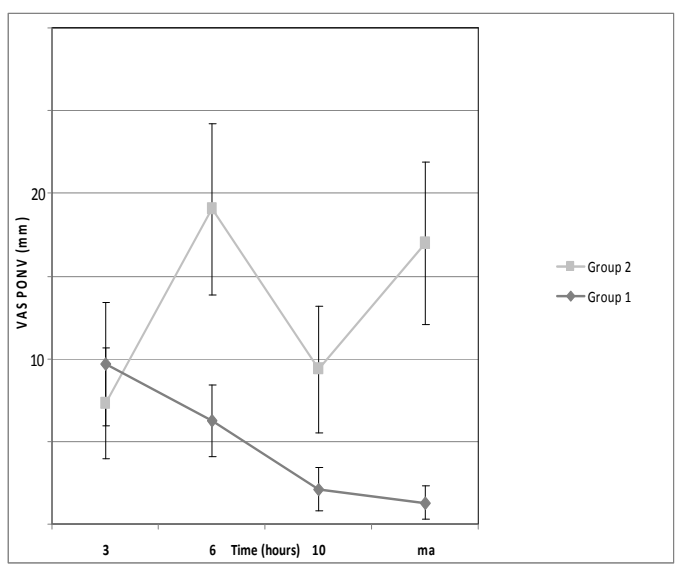

Fig 4. PONV after abdominal hysterectomy. Diagram showing mean VAS of PONV after surgery. Error bars show standard error of the mean. ma $=$ morning the first postoperative day 10 a.m. VAS scores are higher in group 2 than in group 1 the morning after surgery $(\mathrm{p}=0.006)($ Mann-Whitney).

Regarding the questionnaire, $85 \%$ in group 2 compared to $94 \%$ in group 1 indicated that they had received enough analgesia $(p=0.26)$. Furthermore, significantly more patients in group 2 compared to group 1 indicated that they had suffered major nausea (48 versus $18 \%, \mathrm{p}=0.01$ ). Sixty eight percent of the patients in group 2 would recommend the method of analgesia to a close friend who would undergo the same operation compared to $88 \%$ in group $1(\mathrm{p}=0.08)$.

There were no complications except for two wound infections in group 1 . There were no signs of respiratory depression in any of the groups.

\section{Discussion}

The aim of this study was to compare two different regimens of regional anesthetic techniques, one with added and one without morphine, in combination with postoperative administration of ketobemidone regarding to time to discharge, level of postoperative consumption of opioids and PONV. In summary, this study shows that the group that received an intrathecal block with morphine in combination with postoperative intermittent opioid administration consumed less ketobemidone and had less pain and nausea than the group that got an intrathecal block without morphine in combination with PCA. Time to discharge was not different.
The time of discharge or rather hospital stay is an important parameter to measure. However, the concept hospital stay is subjective and dependent on many factors. It could be considered as a surrogate endpoint which is used because it is easy to quantify and can be translated into economical terms. Therefore, in this study we looked for an intervention that could presumably reduce the time to discharge. Spinal administrations of opioids offer segmental analgesia and will increase the analgetic effects of concomitant administered local anesthetics. For optimized intraoperative pain control fentanyl is the best documented opioid while for postoperative pain control morphine would be the best choice due to its longer duration $[9,10]$. However, all intrathecally administered opioids have side effects including pruritus, nausea and vomiting, urinary retention, hypotension and respiratory depression. Although we did not reach a statistical significant difference in time to discharge, there was difference of 16 hours in favor of the group that had morphine in their intrathecal blocks. In all other parameters investigated, however, we reached statistical significance to the advantage of the group given intrathecal blocks with morphine added. The postoperative regimens differed in that group 1 received intermittently nurse administered ketobemidone while group 2 had PCA. PCA should be a much better regimen, although more costly, of administering postoperative pain relief compared to a nurse administered regime. The latter involves an expressed demand by the patient that may not always be fulfilled and also involves a time delay. In spite of this, as shown in Fig 2, the consumption of ketobemidone was significantly lower in group 1 which should point to that these patients had less pain than in the group 2. The self assessed severity of PONV was decreased in group 1 contrast to group 2 . The reason for this is most likely the greater consumption of ketobemidone in the latter group.

Our study has weaknesses. Although this was a randomized, prospective and controlled study it was blinded only to the attending surgeon. Blinding all care providers to intrathecal morphine is not advisable. There were differences between the groups regarding their postoperative analgesic protocols. This was mainly warranted by the fear of respiratory depression in group 1 if they would have had unsupervised access to parenteral 
ketobemidone as the other group had. Most reported cases of respiratory depression are due to the concomitant use of intrathecal morphine and parenteral morphine. This fear was perhaps exaggerated in retrospect and the study would have benefited from giving all patients the same postoperative protocol. Also, the study did not reach statistical difference in time to discharge. The study was powered to find a difference in time to discharge of 17 hours which points to that it is slightly underpowered.

In summary, we conclude that an intrathecal block in combination with postoperative nurse administered ketobemidone improves the wellbeing of the patients and significantly reduces the level of pain and nausea and consumption of postoperative ketobemidone. Furthermore, based on the questionnaire, significantly more patients were satisfied with their analgesia in this group.

\section{References}

1. Gan TJ, Meyer T, Apfel CC, Chung F, Davis PJ, Eubanks S, et al. Consensus guidelines for managing postoperative nausea and vomiting. Anesth Analg. 2003;97(1):62-71.

2. Wilmore DW, Kehlet H. Management of patients in fast track surgery. Bmj. 2001;322:473-6.

3. Watcha MF, White PF. Postoperative nausea and vomiting. Its etiology, treatment, and prevention. Anesthesiology. 1992;77(1):16284.
4. Kenny GN. Risk factors for postoperative nausea and vomiting. Anaesthesia. 1994;49 610.

5. Devys J-M, Mora A, Plaud B, Jayr C, Laplanche A, Raynard B, et al. Intrathecal + PCA morphine improves analgesia during the first $24 \mathrm{hr}$ after major abdominal surgery compared to PCA alone. Can J Anaesth. 2003;50(4):355-61.

6. Kontrimaviciute E, Baublys A, Ivaskevicius J. Postoperative nausea and vomiting in patients undergoing total abdominal hysterectomy under spinal anaesthesia: a randomized study of ondansetron prophylaxis. Eur J Anaesthesiol. 2005;22(7):504-9.

7. Hagle ME, Lehr VT, Brubakken K, Shippee A. Respiratory depression in adult patients with intravenous patient-controlled analgesia. Orthopaedic Nursing. 2004;23(1):18-27.

8. Chang AM, Ip W, Cheung T. Patientcontrolled analgesia versus conventional intramuscular injection: a cost effectiveness analysis. Journal of Advanced Nursing. 2004;46(5):531-41.

9. Mason N, Gondret R, Junca A, Bonnet F. Intrathecal sufentanil and morphine for postthoracotomy pain relief. $\mathrm{Br} \mathrm{J}$ Anaesth. 2001;86(2):236-40.

10. Swart M, Sewell J, Thomas D. Intrathecal morphine for caesarean section: an assessment of pain relief, satisfaction and side-effects. Anaesthesia. 1997;52(4):373-7. 